\title{
Harmonic analysis and hypercomplex function theory in co-dimension one
}

\author{
Helmuth R. Malonek, Isabel Cação ${ }^{1}$, M. Irene Falcão ${ }^{2}$, and Graça Tomaz ${ }^{3}$ \\ 1 CIDMA and Department of Mathematics \\ University of Aveiro \\ 3810-193 Aveiro, Portugal \\ hrmalon@ua.pt \\ 2 CMAT and Department of Mathematics and Applications \\ University of Minho \\ 4710-057 Braga, Portugal \\ 3 CIDMA and Research Unit for Inland Development \\ Department of Mathematics \\ Polytechnic of Guarda \\ 6300-659 Guarda, Portugal
}

\begin{abstract}
Fundamentals of a function theory in co-dimension one for Clifford algebra valued functions over $\mathbb{R}^{n+1}$ are considered. Special attention is given to their origins in analytic properties of holomorphic functions of one and, by some duality reasons, also of several complex variables. Due to algebraic peculiarities caused by non-commutativity of the Clifford product, generalized holomorphic functions are characterized by two different but equivalent properties: on one side by local derivability (existence of a well defined derivative related to co-dimension one) and on the other side by differentiability (existence of a local approximation by linear mappings related to dimension one). As important applications sequences of harmonic Appell polynomials are considered whose definition and explicit analytic representations rely essentially on both dual approaches.
\end{abstract}

Keywords: Clifford algebras, hypercomplex differential forms, hypercomplex derivative, hypercomplex Appell polynomials

\section{Introduction}

In 1968, exactly 50 years ago, E. M. Stein and G. Weiss proved in their seminal paper [1], the "correspondence of irreducible representations of several rotation groups to first order constant coefficient partial differential equations generalizing the Cauchy-Riemann equations." They showed how certain properties of complex one-dimensional function theory extend to solutions of those systems, in particular the fact of being harmonic solutions. In their list of systems one can find a generalized Riesz system [2], the Moisil-Theodoresco system [3], spinor systems as n-dimensional generalization of Dirac's equations [4], Hodge - de Rham equations [5] and special cases of them. But the aim of proving that correspondence 
between representation groups and partial differential equations were merely of qualitative nature and deeply connected with properties of harmonic functions in several real variables. But whereas that paper uses purely real methods, two years later R. Delanghe published On Regular-analytic Functions with Values in a Clifford Algebra [6], thereby extending Fueters results [9] about solutions of the generalized Riesz system from the quaternionic to the general Clifford Algebra case.

Nowadays, function theoretic methods over non-commutative algebras are applied in a big variety of mathematical fields, like for example in potential theory, differential geometry, operator theory, BVP of partial differential equations, analytic number theory, discrete and computational mathematics and their corresponding applications in Sciences and Engineering. For a first introduction, particularly dealing with Maxwell's and Schrödinger's equations the reader should consult Kravchenko [7]. An account about the development of this field until 2000 is given by Delanghe in [8].

But it seems to be remarkable that the first attempt for a systematical development of methods based on Clifford algebras started already 40 years before Delanghe's article [6] and was done by the Swiss mathematician Rudolf Fueter (1880-1950). He initiated around 1930 the foundation of a theory of quaternion valued functions of a quaternion variable ([10]). Being already a worldwide known number theorist, he was interested in such a tool for the development of new analytical methods in Number Theory (c.f. [11]). But soon he recognized that he had found an new approach to generalize the classical complex function theory in a way different from that of holomorphic functions of several complex variables. It was exactly 70 years ago that in the Fall Semester of 1948/49, Fueter gave one of his last lessons on Funktionentheory im Hypercomplexen (Function theory in the hypercomplex) [12]. Since this manuscript of the lessons, still approved by Fueter, were never published as book, it is not well known that it contains as Chapter IV: Funktionentheorie der Clifford'schen Algebren on 70 pages (p.247-317) a complete description of an approach to function theory in Clifford algebras based on generalized Cauchy integral theorems. As far as we know, only in the book [13] one can find a remark that R. Fueter, who is commonly considered only as one of the founders of Quaternionic Analysis (cf. [14]), made already contributions to what was 30 years later coined Clifford Analysis $[15,16]$.

During 50 years, having been guided by the paper [1], the research on qualitative properties of generalized Cauchy-Riemann or Dirac systems continues to rely heavily on methods of representation theory and its relationship to Harmonic Analysis (see $[4,8,15,16]$ ). It legitimates to think about Clifford Analysis as a refinement of Harmonic Analysis. Indeed, in this sense it is a powerful tool for applying those methods to the study of problems in PDE, in particular what concerns their symmetries and the algebraic structures behind (Heisenberg algebras, Lie algebras etc.).

But the aim of this paper is to call attention to a complementary treatment of higher-dimensional generalized Cauchy-Riemann systems, namely to function 
theoretic methods adapted to the approximation and numerical calculation of its solutions, briefly called monogenic functions. The approach we will present is closely connected with the hypercomplex generalization of the Wirtinger calculus (which is the one complex variable case of the Dolbeault calculus in the theory of several complex variables). It is not only the basis for understanding the generalization of the Cauchy approach to holomorphic functions [17] but also clarifies the usefulness of several hypercomplex variables and corresponding differential forms [18-20]. The fact that the conjugate hypercomplex CauchyRiemann operator acts as derivation operator like in the complex case (cf. [21]) confirmed the observation of S. Semmes (1996), that Clifford Analysis can be considered as co-dimension one function theory [22]. Moreover, this non-standard approach opened the way to investigate new analytic, geometric and combinatorial properties of monogenic functions. Even new number theoretic results could be obtained, thereby in some sense coming back to Fueter's motivation for his work [11].

The present paper is a revised and substantially abridged version of [20] including recent results on topics for which the coexistence of both concepts, i.e. hypercomplex derivability as well as hypercomplex differentiability, was essential. After a short introduction to Clifford algebras in Sections 2 and 3, a calculus of alternative hypercomplex differential forms is considered in Section 4. As simple consequences the generalized Stokes' formula as well as a generalized Cauchy integral formula are derived, stressing the relation valid for holomorphic functions in the complex plane, too. At the end of this section the expression of the generalized Cauchy-Riemann operator as areolar derivative in the sense of Pompeiu [23] is given. Naturally, the next Section 5 introduces the concept of hypercomplex derivability again based on alternative differential forms. The analogous expression of the hypercomplex derivative as another areolar derivative in the sense of Pompeiu finishes this section. Section 6 refers to two essential theorems proved in [17] resp. [21] which together establish the complete equivalence of the Cauchy and the Riemann approaches for monogenic functions. As direct consequence of differentiability, Section 7 introduces the local expansion of those hypercomplex functions in power series of several hypercomplex variables almost analogous to the case of multivariate real series expansion. The last Section 8 is dedicated to a first resume of recent results in the theory of generalized sequences of Appell polynomials [24] being a type of application of monogenic functions where all their fundamental properties mentioned before come together.

\section{Clifford algebras}

A finite-dimensional algebra with a unit element over the field of real or complex numbers was formerly known as a hypercomplex system. Clifford algebras are examples of them. They possess an isomorphic representation as elements of a $\left(2^{n} \times 2^{n}\right)$ matrix algebra. The matrix representations of complex numbers and quaternions are well known special cases. 
Clifford algebras as associative non-commutative algebras over the field of real numbers can be defined as follows (for more details and other possibilities see, for instance, [20]).

Definition 1. Let $\left\{e_{0}, e_{1}, \ldots, e_{n}\right\}$ be a ONB of $\mathbb{R}^{n+1}$ subject to the multiplication rules

$$
e_{k} e_{l}+e_{l} e_{k}=-2 \delta_{k l} e_{0}, \quad(k, l=1, \ldots, n),
$$

where $\delta_{i j}$ is the Kronecker symbol. The associative $2^{n}$-dimensional universal Clifford algebra $\mathcal{C} \ell_{0, n}$ over $\mathbb{R}$ with unity is the set of numbers $\alpha \in \mathcal{C} \ell_{0, n}$ written in the form

$$
\alpha=\sum_{A} \alpha_{A} e_{A},
$$

with the basis $\left\{e_{A}: A \subseteq\{1, \ldots, n\}\right\}$ formed by

$$
e_{A}=e_{h_{1}} e_{h_{2}} \ldots e_{h_{r}}, 1 \leq h_{1}<\ldots<h_{r} \leq n, e_{\emptyset}=e_{0}=1
$$

and where the components $\alpha_{A}$ are real numbers. The conjugate element to $\alpha$ is defined by $\bar{\alpha}=\sum_{A} \alpha_{A} \bar{e}_{A}$, where

$$
\bar{e}_{A}=\bar{e}_{h_{r}} \bar{e}_{h_{r-1}} \ldots \bar{e}_{h_{1}} ; \bar{e}_{k}=-e_{k}(k=1, \ldots, n), \bar{e}_{0}=e_{0}=1 .
$$

The norm of $\alpha \in \mathcal{C} \ell_{0, n}$ is defined by $|\alpha|=\left(\sum_{A} \alpha \bar{\alpha}\right)^{1 / 2}=\left(\sum_{A} \alpha_{A}^{2}\right)^{1 / 2}$.

\section{Hypercomplex structures of $\mathbb{R}^{n+1}$}

The theory of complex functions of several complex variables uses for the description of the pre-image set the algebra of complex numbers by combining $2 n$ real variables $\left(x_{1}, \ldots, x_{n}, y_{1}, \ldots, y_{n}\right) \in \mathbb{R}^{2 n}$ to a vector of $n$ complex variables $\left(z_{1}, \ldots, z_{n}\right) \in \mathbb{C}^{n} ; z_{k}=x_{k}+i y_{k}, \quad k=1, \ldots n$. Then it is possible to realize the inverse variable transformation from the complex to the real variables with the help of the vector of their conjugates $\left(\bar{z}_{1}, \ldots, \bar{z}_{n}\right)$ in the form

$$
x_{k}=\frac{1}{2}\left(\bar{z}_{k}+z_{k}\right) \quad \text { and } \quad y_{k}=\frac{i}{2}\left(\bar{z}_{k}-z_{k}\right), \quad k=1, \ldots n .
$$

In such a way $\mathbb{R}^{2 n}$ is endowed with a complex structure by identifying $\mathbb{R}^{2 n}$ with $\mathbb{C}^{n}$, symbolically $\mathbb{R}^{2 n} \cong \mathbb{C}^{n}$. Like in the ordinary case of two real and one complex variables $\left(\mathbb{R}^{2} \cong \mathbb{C}^{1}\right)$ this leads to the reduction of the real dimension $2 n$ to the half, i.e., to $n$, compared to $\mathbb{C}^{n}$. In particular, the local property of complex differentiability of a function $f: \Omega \subset \mathbb{C}^{n} \rightarrow \mathbb{C}$ implies that $f$ may be considered as a function of $z_{k}, \quad k=1, \ldots n$ and not of $\bar{z}_{k}, \quad k=1, \ldots n$. This is due to the fact that it can locally be approximated by a linear mapping (the differential) of the vector $\left(d z_{1}, \ldots d z_{n}\right)$. One important consequence is the representation of $f: \Omega \subset \mathbb{C}^{n} \rightarrow \mathbb{C}$ by multiple power series in $\left(z_{1}, \ldots, z_{n}\right)$ (Weierstrass approach).

If we are looking for a hypercomplex structure of $\mathbb{R}^{n+1}$ in the aforementioned sense, then we can recognize several possibilities for similar procedures. 
I. Paravectors. Let the element $x=\left(x_{0}, x_{1}, \ldots, x_{n}\right)$ of $\mathbb{R}^{n+1}$ be identified with

$$
z=x_{0}+x_{1} e_{1}+\ldots+x_{n} e_{n} \in \mathcal{A}_{n}:=\operatorname{span}_{\mathbb{R}}\left\{1, e_{1}, \ldots, e_{n}\right\}
$$

and called paravector. The conjugate of $z$ is given by

$$
\bar{z}=x_{0}-x_{1} e_{1}-\ldots-x_{n} e_{n}
$$

and its norm is

$$
|z|:=\sqrt{z \bar{z}}=\left(\sum_{i=1}^{n} x_{i}^{2}\right)^{1 / 2}
$$

analogously to the complex case. Therefore each non-zero $z \in \mathcal{A}_{n}$ is invertible and its inverse is $z^{-1}=\frac{\bar{z}}{|z|^{2}}$.

The usual approach to hypercomplex function theory considers $\mathrm{C}_{0, n}$-valued functions of the form $f(z)=\sum_{A} f_{A}(z) e_{A}, \quad f_{A}(z) \in \mathbb{R}$, as mappings

$$
f: \Omega \subset \mathbb{R}^{n+1} \cong \mathcal{A}_{n} \longmapsto \mathcal{C} \ell_{0, n}
$$

Of course, the big advantage of this approach is to deal with only one hypercomplex variable $z$. Compared with the ordinary case of two real and one complex variables $\left(\mathbb{R}^{2} \cong \mathbb{C}^{1}\right)$ it reflects the reduction of the real dimension $(n+1)$ to dimension 1 , i.e., by $n$, compared to $\mathbb{R}^{n+1}$.

II. Several hypercomplex variables. A second hypercomplex structure of $\mathbb{R}^{n+1}$ different from that given by $\mathcal{A}_{n}$ consists in the following isomorphism:

$$
\mathbb{R}^{n+1} \cong \mathcal{H}^{n}=\left\{\mathbf{z}: z_{k}=x_{k}-x_{0} e_{k} ; x_{0}, x_{k} \in \mathbb{R}, k=1, \ldots, n\right\} .
$$

More detailed, this means to take $n$ copies $\mathbb{C}_{k}$ of $\mathbb{C}$ identifying $i \cong e_{k}, \quad(k=$ $1, \ldots, n) ; x_{0} \cong \Re z ; x_{k} \cong \Im z ;$ where $z \in \mathbb{C}$, and let $\mathbb{C}_{k}:=-e_{k} \mathbb{C}$. Then $\mathcal{H}^{n}$ is the cartesian product $\mathcal{H}^{n}:=\mathbb{C}_{1} \times \cdots \times \mathbb{C}_{n}$ and $\mathcal{C} \ell_{0, n}$-valued functions $f(z)=$ $\sum_{A} f_{A}(z) e_{A}$ are considered as mappings

$$
f: \Omega \subset \mathbb{R}^{n+1} \cong \mathcal{H}^{n} \longmapsto \mathcal{C} \ell_{0, n} .
$$

Remark 1. In the following sections we will see that simple algebraic relations allow to consider both approaches as in some sense dual approaches. The qualitatively new point of view is the (not only formal) relationship with $\mathbb{C}^{n}$. Later we will see that one of the most important facts for the usefulness of hypercomplex function theory is its independence from the parity of the underlying real space and its direct relationship to harmonic function theory.

\section{Calculus of alternative hypercomplex differential forms}

In the usual Riemann approach to monogenic functions the hypercomplex form of Stokes' theorem plays a central role. This is due to the fact that for monogenic functions Stokes' theorem can be interpreted as a generalization of Cauchy's 
integral theorem for holomorphic functions in the plane. We will see that the consideration of $\mathcal{H}^{n}$ permits a better insight into the nature of the hypercomplex form of Stokes' theorem, too. Indeed, the hypercomplex form of Stokes' theorem can be derived in a very natural way by using $(n+1)$ hypercomplex differential forms defined by:

$$
d z_{0}=d x_{0}, \quad d z_{k}=-e_{k} d x_{0}+d x_{k} ; k=1, \ldots, n
$$

i.e., in terms of basic real differential 1 -forms. In this way we can see that the $\mathcal{H}^{n}$ approach reflects some formal duality to the case of several complex variables. It is the key to the notion of hypercomplex derivative and also reveals the role of the conjugated Cauchy-Riemann operator as derivation operator. Therefore we define the outer product of the basic differential forms (1) in the following way:

Definition 2. The product of two basic hypercomplex 1-forms is defined by

$$
\begin{aligned}
d z_{k} \wedge d z_{l} & =\left(-e_{k} d x_{0}+d x_{k}\right) \wedge\left(-e_{l} d x_{0}+d x_{l}\right) \\
& =d x_{k} \wedge d x_{l}-e_{k} d x_{0} \wedge d x_{l}-e_{l} d x_{k} \wedge d x_{0} \\
d z_{k} \wedge d z_{0} & =\left(-e_{k} d x_{0}+d x_{k}\right) \wedge d x_{0}=d x_{k} \wedge d x_{0} ; \quad k, l=1, \ldots, n
\end{aligned}
$$

A basic hypercomplex $p$-form is the outer product of $p$ different basic hypercomplex 1-forms.

The general form of a basic hypercomplex $p$-form can easily be written with the help of a multi-index $\nu=\left(\nu_{0}, \nu_{1}, \ldots, \nu_{p-1}\right), 0 \leq \nu_{0}<\nu_{1}<\cdots<\nu_{p-1} \leq n$ and relies on the fact (property 1 below) that the set of basic hypercomplex 1-forms is an alternative outer algebra (exactly as in the case of real differential forms). Therefore every basic $p$-form $\omega_{p} \in \bigwedge_{\mathcal{A}_{n}}^{p}$ can be written in a unique way as

$$
\omega_{p}=d z_{\nu_{0}} \wedge d z_{\nu_{1}} \wedge \ldots \wedge d z_{\nu_{p-1}}=: d z_{\nu}
$$

The verification of the following properties is straightforward.

1. The outer product is alternative, i.e.,

$$
d z_{k} \wedge d z_{l}=-d z_{l} \wedge d z_{k}, \quad k \neq l, \quad d z_{k} \wedge d z_{k}=0
$$

2. If the notation $d \hat{x}_{m}(m=0, \ldots, n)$ means that in the ordered outer product of the 1-forms $d x_{k}(k=0, \ldots, n)$ the factor $d x_{m}$ is absent, then the hypercomplex surface element in $\mathbb{R}^{n+1}$ is given by $d \sigma_{(n)}=d \hat{x}_{0}-e_{1} d \hat{x}_{1}+\cdots+$ $(-1)^{n} e_{n} d \hat{x}_{n}$ (cf. [16]). In terms of (1) there holds

$$
d \sigma_{(n)}=d z_{1} \wedge \cdots \wedge d z_{n}
$$

3. The volume element $d V$ in $\mathbb{R}^{n+1}$ has in terms of (1) the simple expression

$$
d V=d z_{0} \wedge d \sigma_{(n)}=(-1)^{n} d \sigma_{(n)} \wedge d z_{0}=d z_{0} \wedge d z_{1} \wedge \cdots \wedge d z_{n}
$$


4. With the usual notation $\partial_{k}=\frac{\partial}{\partial x_{k}}$, let $d f=\partial_{0} f d x_{0}+\partial_{1} f d x_{1} \cdots+\partial_{n} f d x_{n}$ be the differential of $f \in \mathcal{C}^{1}\left(\mathbb{R}^{n+1}, \Omega\right)$. By using (1), the right (resp. left) hypercomplex form of the differential of $f$ has the form:

$$
\begin{aligned}
& d f=(f D) d z_{0}+\partial_{1} f d z_{1}+\cdots+\partial_{n} f d z_{n} \text { resp. } \\
& d f=d z_{0}(D f)+d z_{1} \partial_{1} f+\cdots+d z_{n} \partial_{n} f,
\end{aligned}
$$

where

$$
D=\partial_{0}+e_{1} \partial_{1}+\cdots+e_{n} \partial_{n}
$$

is the so-called generalized Cauchy-Riemann operator.

If $\bar{D}=\partial_{0}-e_{1} \partial_{1}-\cdots-e_{n} \partial_{n}$ denotes the conjugate operator to $D$ then $D \bar{D}=\bar{D} D=\Delta$, factorizing the Laplace operator like in the complex case.

5 . Consider the hypercomplex $(n-1)$-form

$$
d \sigma_{(n-1)}:=-e_{1} d \hat{x}_{0,1}+e_{2} d \hat{x}_{0,2}+\cdots+(-1)^{n} e_{n} d \hat{x}_{0, n},
$$

where $d \hat{x}_{0, m} \quad(m=1, \ldots, n)$ stands for the ordered outer product of the 1 -forms $d x_{k}(k=0, \ldots, n)$ where the factors $d x_{0}$ and $d x_{m}$ are absent. Then for $n=1$ the constant 0 - form $d \sigma_{(0)}=-e_{1} \cong-i$ is obtained, i.e., exactly the factor that is necessary to pass from $z \in \mathbb{C}$ to $z_{1}=-i z \in \mathbb{C}_{1}$. The designation of this $(n-1)$ - form by $d \sigma_{(n-1)}$ is based in the following fact. The 1-form of the vector-part of $d z$, i.e.,

$$
\text { Vec } d z=e_{1} d x_{1}+\ldots+e_{n} d x_{n}
$$

can be considered as related to a hyperplane $x_{0}=c=$ const. Then $d \sigma_{(n-1)}$ is just the corresponding surface-element with respect to the effective variables $\left(x_{1}, \ldots, x_{n}\right)$.

Now let $\nu=\left(\nu_{0}, \nu_{1}, \ldots, \nu_{p-1}\right), 0 \leq \nu_{0}<\nu_{1}<\cdots<\nu_{p-1} \leq n$, be a multi-index, and for a given set $f_{\nu}=f_{\nu}(z)$ of $\left(\begin{array}{c}n+1 \\ p\end{array}\right) \mathcal{C} \ell_{0, n}$-valued continuously differentiable functions in $\Omega$, consider the forms $\omega_{p}=\sum_{\nu} d z_{\nu} f_{\nu}(z)$, resp. $\omega_{p}=\sum_{\nu} f_{\nu}(z) d z_{\nu}$. In the case of a left form we have:

Definition 3. Let $\omega_{p}=\sum_{\nu} d z_{\nu} f_{\nu}(z)$ be a continuously real differentiable left $p$-form on $\Omega$. Then its (outer) derivative $d \omega_{p}$ is defined as the $(p+1)$-form

$$
d \omega_{p}=\sum_{\nu}(-1)^{p} d z_{\nu} \wedge d f_{\nu}(z)
$$

where $d f_{\nu}$ is the differential of $f_{\nu}$, i.e., the outer derivative of the 0 -form $f_{\nu}$.

For right $p$-forms or two-sided $p$-forms $\omega_{p}$ (i.e., having $\mathcal{C} \ell_{0, n}$-valued coefficients on both sides) the definition of $d \omega_{p}$ is straightforward.

The use of alternative hypercomplex differential forms permits a very lucid deduction of the hypercomplex form of Stokes' integral theorem as well as the 
generalization of Cauchy's theorem. Therefore let $\omega=f(z) d \sigma_{(n)}$ be a hypercomplex differential $n$ - form built in analogy to the real case as a product of the surface element $d \sigma_{(n)}$ in $\mathbb{R}^{n+1}$, i.e. (2), with a function $f \in \mathcal{C}^{1}\left(\mathbb{R}^{n+1}, \Omega\right)$. The general Stokes' theorem in the form

$$
\int_{\partial \Omega} \omega=\int_{\Omega} d \omega
$$

applied to a $(n+1)$-dimensional positively oriented domain $\Omega$ implies together with the Definition 3 of the outer derivative and with (3) that

$$
\int_{\partial \Omega} d \sigma_{(n)} f(z)=\int_{\Omega}(-1)^{n} d \sigma_{(n)} \wedge d f=\int_{\Omega}(-1)^{n} d \sigma_{(n)} \wedge d z_{0} D f=\int_{\Omega} D f d V .
$$

The following theorem shows that the generalized Cauchy-Riemann operator $D$ can be characterized as an areolar derivative in the sense of Pompeiu. The concept of areolar derivative in the sense of Pompeiu originated from [23] and has been discussed in the hypercomplex context in [13,20,25].

Theorem 1. Let $\mathbf{z}_{\star}$ be a fixed point in a positively oriented differentiable and contractible domain $\Omega \subset \mathcal{H}^{n}$ with smooth boundary. Consider a regular sequence of subdomains $\left\{\Omega_{n}\right\}$ which is shrinking to $\mathbf{z}_{\star}$ if $n$ tends to infinity and whereby $\mathbf{z}_{\star}$ belongs to all $\Omega_{n}$. For a real differentiable function $f$ defined in $\Omega$ holds

$$
(f D)\left(\mathbf{z}_{\star}\right)=\lim _{n \rightarrow \infty} \frac{1}{\operatorname{mes} \Omega_{n}} \int_{\partial \Omega_{n}} f(z) d z_{1} \wedge \cdots \wedge d z_{n}
$$

i.e. the (right) generalized Cauchy-Riemann operator $D$ is a (right) generalized areolar derivative in the sense of Pompeiu of $f=f(\mathbf{z})$ over $\Omega$ at $\mathbf{z}_{\star}$.

Remember that in our notation, using (3),

$$
\operatorname{mes} \Omega_{n}=\int_{\Omega_{n}} d V=\int_{\Omega_{n}} d z_{0} \wedge d z_{1} \wedge \cdots \wedge d z_{n} .
$$

The proof of this theorem relies on the application of the hypercomplex form of Stokes' theorem and the mean value property (c.f. [20]).

Only one step is left for obtaining a generalized Cauchy's integral formula. Therefore we mention

Definition 4. Let $f=f(\mathbf{z})$ be continuously real differentiable in an open set $\Omega \subset \mathbb{R}^{n+1} \cong \mathcal{H}^{n} \cong \mathcal{A}_{n}$. Then $f$ is called left (resp. right) monogenic in $\Omega$, if and only if $D f=0(f D=0)$.

The components $f_{A}(\mathbf{z})$ of a monogenic function $f(\mathbf{z})$ are real harmonic functions not only pluri-harmonic like in the case of several complex variables ("refinement" of harmonic function theory).

The designation of left (resp. right) monogenic functions which we are also using has historically been introduced in [16]. For stressing more the complete 
coincidence with the situation in the complex case, recently in [13] and other papers $f$ is called (Clifford) holomorphic.

Cauchy's integral formula follows immediately from (4): If $f$ is (left) monogenic then $\omega=d \sigma_{(n)} f(\mathbf{z})$ is closed, i.e.

$$
\int_{\partial \Omega} d \sigma_{(n)} f(\mathbf{z})=0
$$

Now we are able to introduce the already announced notion of hypercomplex derivability and the definition of the hypercomplex derivative (c.f. [21]).

\section{Hypercomplex derivability}

The notion of left (L-) or right (R-) hypercomplex derivability relies on the intrinsic relations between two forms of degree $n$ (more concretely on $d \sigma_{(n)}$ and $d\left(d \sigma_{(n-1)} f\right)$ or $\left.d\left(f d \sigma_{(n-1)}\right)\right)$ generalizing an idea of [26].

Definition 5. A function $f: \mathcal{H}^{n} \mapsto \mathcal{C} \ell_{0, n}$ is $L$ - (R-) derivable at $\mathbf{z} \in \mathcal{H}^{n}$ if it is real differentiable at $\mathbf{z}$ and there exists $A_{f, L}(\mathbf{z})\left(A_{f, R}(\mathbf{z})\right) \in \mathcal{C} \ell_{0, n}$ such that

$$
d\left(d \sigma_{(n-1)} f\right)=d \sigma_{(n)} A_{f, L}(\mathbf{z}) \text { resp. } d\left(f d \sigma_{(n-1)}\right)=A_{f, R}(\mathbf{z}) d \sigma_{(n)} .
$$

$A_{f, L}(\mathbf{z})\left(A_{f, R}(\mathbf{z})\right)$ are called the left and right derivative of $f$ at $\mathbf{z}$, respectively.

In [21] for the first time could be proven that the conjugate Cauchy-Riemann operator, more concretely $\frac{1}{2} \bar{D}$, acts like a hypercomplex derivation operator as one should expect in analogy to the complex case, since $A_{f, L}(\mathbf{z})=\frac{1}{2} \bar{D} f$, resp. $A_{f, R}(\mathbf{z})=\frac{1}{2} f \bar{D}$. Moreover, with this definition one has a generalization of the classical Cauchy approach which relies directly on a limit process like in the classical complex case. In analogy to Theorem 1 one has also its expression in form of an areolar derivative in the sense of Pompeiu. In the left (and analogously in the right) case we have

Theorem 2. Let $\mathcal{S} \subset \Omega$ be an oriented differentiable $n$-dimensional hypersurface with boundary $\partial \mathcal{S}_{m}$ and $\mathbf{z}^{*}$ be a fixed point in $\mathcal{S}$. Consider a sequence of subdomains $\left\{\mathcal{S}_{m}\right\}$ which is shrinking to $\mathbf{z}^{*}$ if $m \rightarrow \infty$. Suppose now that the function $f$ is left monogenic in $\Omega$, i.e. $D f=0$. Then the left hypercomplex derivative $\frac{1}{2} \bar{D}$ is an areolar derivative in the sense of Pompeiu of the form

$$
\frac{1}{2} \bar{D} f=\lim _{m \rightarrow \infty}\left[\int_{\mathcal{S}_{m}} d \sigma_{(n)}\right]^{-1} \int_{\partial \mathcal{S}_{m}}\left(d \sigma_{(n-1)} f\right) .
$$

Moreover, following [21], we have

Theorem 3. Consider $f=f(\mathbf{z})$ with $\mathbf{z} \in \Omega \subset \mathcal{H}^{n}$. Then $f$ is left (resp. right) monogenic in $\mathbf{z}$ iff $f$ has in $\mathbf{z}$ a uniquely defined hypercomplex areolar derivative $f_{L}^{\prime}=\frac{1}{2} \bar{D} f ;\left(f_{R}^{\prime}=\frac{1}{2} f \bar{D}\right)$ in the sense of Pompeiu. 
For example, for the left case, $\frac{1}{2} \bar{D} f=\frac{1}{2}\left(\partial_{0} f-e_{1} \partial_{1} f-\cdots-e_{n} \partial_{n} f\right)=\partial_{0} f$ due to the fact that $D f=\partial_{0} f+e_{1} \partial_{1} f+\cdots+e_{n} \partial_{n} f=0$. Obviously, the hypercomplex derivative of a monogenic function $f$ is a differential coefficient between two differential forms (cf. [26]), this time not of degree 1 as usual, but of degree $n$ (which is, of course, the same in the simplest case $n=1$, i.e., in the complex plane case). Notice also the natural presence of the factor $1 / 2$ on $\bar{D}$ like in the case of Wirtinger's complex partial derivative $\partial_{z}=\frac{1}{2}\left(\partial_{x}-i \partial_{y}\right)$. Several formulas would become more symmetric and compatible with the complex case, if one would use this coefficient also in the hypercomplex case. For simplicity and from now on we use the notation $\partial:=\frac{1}{2} \bar{D}$.

\section{Hypercomplex differentiability}

The adequate definition of hypercomplex differentiability as generalization of complex differentiability remained an unsolved problem until the end of the 80ties of the last century (c.f. [27]). Based on the use of the hypercomplex structure expressed by $\mathbb{R}^{n+1} \cong \mathcal{H}^{n}$ and the fact that differentiability is equivalent with the possibility of local linearization of the increment of the considered function, we have the following

Definition 6. Let $f$ be a continuous mapping from a neighborhood of $\mathbf{z}_{*} \in \mathcal{H}^{n}$ into $\mathcal{C} \ell_{0, n}$. Then $f$ is called left hypercomplex differentiable (resp. right hypercomplex differentiable) at $\mathbf{z}_{*}$ if there exists a left (resp. right) $\mathcal{C} \ell_{0, n}$-linear mapping $\ell$ such that

$$
\lim _{\Delta \mathbf{z} \rightarrow 0} \frac{\left|f\left(\mathbf{z}_{*}+\Delta \mathbf{z}\right)-f\left(\mathbf{z}_{*}\right)-\ell(\Delta \mathbf{z})\right|}{\|\Delta \mathbf{z}\|}=0 .
$$

We say that a function $f$ is hypercomplex differentiable in $\Omega \subset \mathbb{R}^{n+1} \cong \mathcal{H}^{n}$ if it is hypercomplex differentiable at all points of $\Omega$ (c.f. [1'7]).

Moreover, it has been shown in [17], that the equivalence of the concept of hypercomplex differentiability and monogeneity is guaranteed. Analogously to Theorem 3 the following theorem is valid.

Theorem 4. Let $f=f(\mathbf{z})$ be continuously real differentiable in an open set $\Omega \subset \mathcal{H}^{n}$. Then $f$ is hypercomplex $L$ - $(R-)$ differentiable in $\Omega$, if and only if $D f=0(f D=0)$ in $\Omega$, i.e. if $f$ is left (resp. right) monogenic.

Again, but now through the property of hypercomplex differentiability the Cauchy approach to holomorphic functions is generalized. In [28], which discussed the impossibility of adequate generalizations of Cauchy's as well as Weierstrass' approaches (by the concept of convergent power series) to holomorphic functions in the higher-dimensional hypercomplex setting, this approach has been overseen. Both theorems, Theorem 3 together with Theorem 4, show that hypercomplex derivability, hypercomplex differentiability and monogeneity of a hypercomplex function $f: \Omega \subset \mathbb{R}^{n+1} \cong \mathcal{H}^{n} \longmapsto \mathcal{C} \ell_{0, n}$ are equivalent. 


\section{Generalized power series}

The aim of this section is to show that the $\mathcal{H}^{n}$-approach to monogenic functions leads in a very natural and direct way to power series in several hypercomplex variables (Weierstrass approach). For instance, no auxiliary application of Legendre or Gegenbauer polynomials (cf. $[14,16]$ ) is needed. In general, the non-commutative multiplication in Clifford algebras causes many difficulties in hypercomplex function theory. But the systematical use of $n$ hypercomplex variables allows a deeper structural insight from the beginning and simplifies essentially the calculations, particularly when dealing with monogenic polynomials. For more details see [20]. We begin by illustrating some aforementioned facts in relation to the classical complex case thereby also calling attention to important differences to the hypercomplex case.

1. Consider an open set $\Omega \subset \mathbb{R}^{3}$ and a continuously differentiable vector field (the velocity field of a flow) $\mathbf{g}=\left(g_{0}, g_{1}, g_{2}\right)$ on $\Omega$. Then the flow of a noncompressible fluid without sources nor sinks is the solution of a Riesz system (particular case of the Riesz system considered in [1]), namely

$$
\left\{\begin{array}{c}
\operatorname{div} \mathbf{g}=0 \\
\text { curl } \mathbf{g}=0
\end{array} .\right.
$$

In the equivalent hypercomplex setting we consider a paravector valued function $f=f_{0}+f_{1} e_{1}+f_{2} e_{2}$ where $\left(f_{0}, f_{1}, f_{2}\right):=\left(g_{0},-g_{1},-g_{2}\right)$. Then the Riesz system can be written with respect to $f$ in the compact form as

$$
D f=0 \text { or } f D=0
$$

and represents obviously a generalized Cauchy-Riemann system in $\mathbb{R}^{3}$.

2. The hypercomplex variables

$$
f_{k}(z)=z_{k}:=x_{k}-x_{0} e_{k}=-\frac{1}{2}\left[z e_{k}+z e_{k}\right], \quad(k=1, \ldots, n)
$$

are left- and right-monogenic functions, also called totally regular variables (c.f. $[6,14])$.

3. The identity function $f(z)=z \in \mathcal{A}_{n}$ is not monogenic unless $n=1$ (the classical complex case), since $D f=f D=1-n$.

4. Powers of $z$, i.e. $f(z)=z^{n}$ and simple products of the totally regular variables like $z_{j} \cdot z_{k}, \quad j \neq k$, are not monogenic. This and the previous property show that it is not possible to deal in the same way as usual with the nonmonogenic ordinary power of the underlying variable $z \in \mathcal{A}_{n}$.

5. Symmetric products of the totally regular variables in the form

$$
z_{j} \times z_{k}:=\frac{1}{2}\left[z_{j} z_{k}+z_{k} z_{j}\right]=x_{j} x_{k}-x_{0} x_{k} e_{j}-x_{0} x_{j} e_{k}
$$

are left- and right-monogenic. More general, it has been proven in [29], that if $\nu=\left(\nu_{1}, \ldots, \nu_{n}\right)$ is a multi-index, all homogeneous monogenic polynomials 
of degree $|\nu|=k$ can be obtained as linear combinations (from the left or from the right) of generalized powers given in the form

$$
\begin{aligned}
\mathbf{z}^{\nu}:=z_{1}^{\nu_{1}} \times \cdots \times z_{n}^{\nu_{n}} & =\underbrace{z_{1} \times \cdots \times z_{1}}_{\nu_{1}} \times \cdots \times \underbrace{z_{n} \times \cdots \times z_{n}}_{\nu_{n}} \\
& =\frac{1}{k !} \sum_{\pi\left(i_{1}, \ldots, i_{k}\right)} z_{i_{1}} \cdots z_{i_{k}},
\end{aligned}
$$

where the sum is taken over all permutations of $\left\{i_{1}, \ldots, i_{k}\right\} \subseteq\{1, \ldots, n\}$ and $z_{j}=x_{j}-x_{0} e_{j}, j=1, \ldots, n$.

Moreover, all functions of the form $f(z)=\mathbf{z}^{\nu}$, are left and right monogenic and $C l_{0, n}$ - linear independent. Therefore they can be used as basis for generalized power series. Following [29] it has been shown that the generalized power series of the form

$$
P(\mathbf{z})=\sum_{k=0}^{\infty}\left(\sum_{|\nu|=k} \mathbf{z}^{\nu} c_{\nu}\right), c_{\nu} \in C l_{0, n}
$$

generates in the neighborhood of the origin a monogenic from the left function $f(\mathbf{z})$ and coincides in the interior of its domain of convergence with the Taylor series of $f(\mathbf{z})$, i.e, in a neighborhood of the origin we have

$$
f(\mathbf{z})=\sum_{k=0}^{\infty} \frac{1}{k !}\left(\sum_{|\nu|=k} \mathbf{z}^{\nu}\left(\begin{array}{l}
k \\
\nu
\end{array}\right) \frac{\partial^{|\nu|} f(\mathbf{0})}{\partial \mathbf{x}^{\nu}}\right),
$$

where $\mathbf{x}=\left(x_{1}, \ldots, x_{n}\right)$. Analogously monogenic from the right power series can be considered as we will do in the sequel.

By using classical methods of estimation the following theorem can be proved:

Theorem 5 ([20]). Let $P(\mathbf{a}, \mathbf{z})$ be a given right power series around $\mathbf{a} \in \mathcal{H}^{n}$. If $P(\mathbf{a}, \mathbf{z})$ converges in some polycylindric domain of the form

$\mathcal{U}(\mathbf{r}, \mathbf{a})=\left\{\mathbf{z} \in \mathcal{H}^{n}:\left|z_{k}-a_{k}\right|=\left(\left(x_{0}-\alpha_{0}\right)^{2}+\left(x_{k}-\alpha_{k}\right)^{2}\right)^{1 / 2}<r_{k}, k=1, \ldots, n\right\}$, then $P(\mathbf{a}, \mathbf{z})$ is right hypercomplex differentiable in $\mathcal{U}(\mathbf{r}, \mathbf{a})$ and the partial derivatives with respect to $x_{k}$ are obtained by formal differentiation as

$$
\frac{\partial P(\mathbf{z})}{\partial x_{k}}=\sum_{\mu} c_{\mu} \mu_{k}(\mathbf{z}-\mathbf{a})^{\mu-\tau_{k}}
$$

where $\tau_{k}$ is the multi-index with 1 at place $k$ and zero otherwise.

Formula (6) shows that the derivatives also represent monogenic functions in the same domain of convergence. By induction follows 
Theorem 6. Every $R_{-}\left(L_{-}\right)$generalized power series is infinitely $R_{-}\left(L_{-}\right)$hypercomplex differentiable inside the domain of convergence.

Calculating the partial derivatives of the generalized powers $(\mathbf{z}-\mathbf{a})^{\mu}$ in $\mathbf{z}=\mathbf{a}$ leads to

$$
\frac{\partial^{|\nu|}}{\partial \mathbf{x}^{\nu}}(\mathbf{z}-\mathbf{a})^{\mu}= \begin{cases}\mu !, & \text { if } \nu=\mu \\ 0, & \text { if } \nu \neq \mu\end{cases}
$$

and this implies

Theorem 7. Every convergent R-power series generates in the interior of its domain of convergence a monogenic function $f(\mathbf{z})$ and coincides there with the Taylor series of $f(\mathbf{z})$, i.e., in a neighborhood of $\mathbf{z}=\mathbf{a}$ we have

$$
f(\mathbf{z})=\sum_{\mu} \frac{1}{\mu !} \frac{\partial^{|\mu|} f(\mathbf{a})}{\partial \mathbf{x}^{\mu}}(\mathbf{z}-\mathbf{a})^{\mu}
$$

(analogously for L-series; there the coefficients are on the right side of the powers).

In view of the unique determination of the coefficients of the Taylor series of a monogenic function we can formulate the uniqueness theorem for generalized Taylor series:

Theorem 8. If the coefficients of two generalized $R-(L-)$ Taylor series coincide in an arbitrarily small neighborhood of the common point of development $\mathbf{a}$ than they coincide identically.

The uniqueness theorem is the basis for the Cauchy-Kowalewskaya extension of a real-analytic $\mathcal{C} \ell_{0, n}$-valued function in $\mathbb{R}^{n}$ :

Theorem 9. Let $f(\mathbf{x})$ be real-analytic in the parallelepiped

$$
\mathcal{V}(\mathbf{r})=\left\{\mathbf{x} \in \mathbb{R}^{n}:\left|x_{k}\right|<r_{k}, k=1, \ldots, n\right\} .
$$

Then an analytic continuation of $f$ to an $R_{-}^{-}\left(L_{-}\right)$monogenic function in

$$
\mathcal{U}(\mathbf{r})=\left\{\mathbf{z} \in \mathcal{H}^{n}:\left|z_{k}\right|<r_{k}, k=1, \ldots, n\right\} .
$$

is given in a unique way by the function

$$
f_{R}^{*}(\mathbf{z})=\sum_{|\mu|=0} \frac{1}{\mu !} \frac{\partial^{|\mu|} f(0)}{\partial \mathbf{x}^{\mu}} \mathbf{z}^{\mu} \text { resp. } f_{L}^{*}(\mathbf{z})=\sum_{\mu} \mathbf{z}^{\mu} \frac{1}{\mu !} \frac{\partial^{|\mu|} f(0)}{\partial \mathbf{x}^{\mu}}
$$

and we have

$$
\left.f_{R}^{*}(\mathbf{z})\right|_{x_{0}=0}=\left.f_{L}^{*}(\mathbf{z})\right|_{x_{0}=0}=\left.f^{*}(\mathbf{z})\right|_{\mathbb{R}^{n}}=f(\mathbf{x}) .
$$


Proof. Inside of $\mathcal{V}(\mathbf{r})$ the function $f(\mathbf{x})$ has the Taylor series representation

$$
f(\mathbf{x})=\sum_{|\mu|=0} \frac{1}{\mu !} \frac{\partial^{|\mu|} f(0)}{\partial \mathbf{x}^{\mu}} \mathbf{x}^{\mu}
$$

and

$$
\left.f_{R}^{*}(\mathbf{z})\right|_{x_{0}=0}=\left.f_{L}^{*}(\mathbf{z})\right|_{x_{0}=0}=\left.f^{*}(\mathbf{z})\right|_{\mathbb{R}^{n}}=f(\mathbf{x})
$$

becomes obvious. The convergence of $(7)$ in $\mathcal{U}(\mathbf{r})$ is guaranteed by the convergence of (8) in $\mathcal{V}(\mathbf{r})$ and the uniqueness follows from the uniqueness theorem for generalized power series.

The $f_{R}^{*}(\mathbf{z})$ resp. $f_{L}^{*}(\mathbf{z})$ are called the right (resp. left) Cauchy-Kowalewskaya extension (CK-extension) of $f$. In fact, Theorem 9 together with property 5 mentioned in the begin of this section, immediately indicates the way of passing from a power series in $\mathbf{x}$, i.e. given in $\mathbb{R}^{n}$, to its monogenic continuation in $\mathcal{H}^{n}$ as function of $\mathbf{z}$ simply by substituting $\mathbf{x}$ by $\mathbf{z}$ and changing at the same time the ordinary product to the symmetric product (5).

\section{An application: Hypercomplex Appell polynomials}

\subsection{Definition and some properties}

In 1880, Appell [24] considered sequences of polynomials of degree $k$ of one real variable, $\left(p_{k}(x)\right)_{k \geq 0}$, satisfying the recurrence relations

$$
p_{k}^{\prime}(x)=k p_{k-1}(x), k=1,2, \ldots,
$$

where $p_{0}(x)$ is a non-zero constant.

Nowadays, any such sequence is called an Appell sequence, its members are called Appell polynomials and (9) is usually referred as Appell property. Of course, the basic idea is that the Appell polynomials behave like power-law functions under the differentiation operation. The classical examples of Appell polynomials besides the monomials $\left(x^{k}\right)_{k \geq 0}, x \in \mathbb{R}$ are the Bernoulli, Euler and Hermite polynomials.

The following equivalent conditions characterizing Appell polynomials underpin the different approaches that have been developed to deal with real Appell sequences as well as their generalizations (see e.g. [30-32]).

Theorem 10. Let $\left(p_{k}(x)\right)_{k \geq 0}$ be a sequence of polynomials of one real variable. Then the following conditions are equivalent:

1. $\left(p_{k}(x)\right)_{k \geq 0}$ is an Appell sequence;

2. $\left(p_{k}(x)\right)_{k \geq 0}$ satisfies

$$
p_{k}(x+y)=\sum_{j=0}^{k}\left(\begin{array}{l}
k \\
j
\end{array}\right) x^{k-j} p_{j}(y)
$$


3. There exists $\left(a_{k}\right)_{k \geq 0}$, with $a_{0} \neq 0$, such that

$$
p_{k}(x)=\sum_{j=0}^{k}\left(\begin{array}{l}
k \\
j
\end{array}\right) a_{j} x^{k-j}
$$

In hypercomplex analysis context, the hypercomplex derivative of a monogenic function is the key player in the generalization of Appell's concept of powerlike polynomial sequences (9) to higher dimensions. This generalization was first introduced in [33] by using several hypercomplex variables (see Theorem 13) and reads as follows.

Definition 7. A sequence of $\mathcal{A}_{n}$-valued monogenic polynomials $\left(\mathcal{F}_{k}(x)\right)_{k \geq 0}$ is called an Appell sequence, if $\mathcal{F}_{k}(x)$ is of exact degree $k$ and

$$
\partial \mathcal{F}_{k}(x)=k \mathcal{F}_{k-1}(x), \quad x \in \mathcal{A}_{n}, \quad k=1,2, \ldots,
$$

where $\mathcal{F}_{0}(x)$ is a non-zero constant.

Based on this concept, it is possible to obtain a binomial-type identity for hypercomplex Appell sequences, which extend the identity (10) to the hypercomplex case [34, Theorem 1].

Theorem 11. A monogenic polynomial sequence $\left(P_{k}(x)\right)_{k \geq 0}$ is an Appell sequence if and only if it satisfies

$$
P_{k}(x)=P_{k}\left(x_{0}+\underline{x}\right)=\sum_{j=0}^{k}\left(\begin{array}{c}
k \\
j
\end{array}\right) x_{0}^{k-j} P_{j}(\underline{x}) .
$$

Hypercomplex Appell polynomials have received a lot of attention in the last decade. They have been studied in detail in several papers by different authors and various applications have been considered [33-41]. In what follows we focus on a class of polynomials of the form

$$
P_{k}(x)=\sum_{s=0}^{k}\left(\begin{array}{l}
k \\
s
\end{array}\right) c_{s}(n) x_{0}^{k-s} \underline{x}^{s}
$$

with coefficients $c_{s}(n) \in \mathbb{R}$ and $c_{0}(n) \neq 0$, for all $n$. For such class of polynomials we can derive an hypercomplex analogue of (11) (see [39, Theorem 2] for details). Moreover, we have (cf.[39, 42]):

Theorem 12. Polynomials $P_{k}(x)$ of the form (13) are monogenic if and only if

$$
c_{k}(n)=\frac{\left(\frac{1}{2}\right)_{\left\lfloor\frac{k+1}{2}\right\rfloor}}{\left(\frac{n}{2}\right)_{\left\lfloor\frac{k+1}{2}\right\rfloor}} c_{0}(n),
$$

where $a_{(r)}=\frac{\Gamma(a+r)}{\Gamma(a)},(r=1,2, \ldots)$ denotes the Pochhammer symbol, with $a_{(0)}=1$. In such cases, $\left(P_{k}(x)\right)_{k \geq 0}$ is an Appell sequence 
Polynomials of the form (13)-(14) with the normalization $c_{0}(n)=1$ have been referred as Standard Appell Polynomials (SAP) in [43] and denoted by $\mathcal{P}_{k}^{n}(x)$. It is clear that this initial value can be changed to any real or hypercomplex constant different from zero. In [44] the concept of Appell sequence was generalized further by considering as first term an arbitrary generalized polynomial constant of a fixed degree.

We stress the fact that $\mathcal{P}_{k}^{1}(x)=\left(x_{0}+e_{1} x_{1}\right)^{k}$ are the usual powers of the holomorphic variable $z=x_{0}+e_{1} x_{1}$ (with the usual identification of $e_{1}$ with the complex imaginary unit). Moreover, real values of $x$ lead to $\mathcal{P}_{k}^{n}\left(x_{0}\right)=x_{0}^{k}$, while for $x=\underline{x}$, we obtain the essential property which characterizes the difference to the complex case $\mathcal{P}_{k}^{n}(\underline{x})=c_{k}(n) \underline{x}^{k}$. One straightforward approach to construct SAP is to consider the CK-extension of the powers $c_{k}(n) \underline{x}^{k}$, which leads to the following equivalent representation of $\mathcal{P}_{k}^{n}(x)$ in terms of generalized powers (5).

Theorem 13. The SAP can be expressed as

$$
\mathbf{P}_{k}(\mathbf{z})=c_{k}(n) \sum_{|\nu|=k} z_{1}^{\nu_{1}} \times \cdots \times z_{n}^{\nu_{n}}\left(\begin{array}{l}
k \\
\nu
\end{array}\right) e_{1}^{\nu_{1}} \times \cdots \times e_{n}^{\nu_{n}},
$$

Since $\mathcal{P}_{k}^{n}(1)=\mathbf{P}_{k}\left(-e_{1},-e_{2}, \ldots,-e_{n}\right)=1$, Theorems 12-13 allow to derive the following relation involving the generators of the algebra.

$$
\sum_{|\nu|=k}(-1)^{k}\left(\begin{array}{l}
k \\
\nu
\end{array}\right)\left(e_{1}^{\nu_{1}} \times \cdots \times e_{n}^{\nu_{n}}\right)^{2}=\frac{\left(\frac{n}{2}\right)_{\left\lfloor\frac{k+1}{2}\right\rfloor}}{\left(\frac{1}{2}\right)_{\left\lfloor\frac{k+1}{2}\right\rfloor}}
$$

This identity is one among many others nice relations that have been obtained through the use of the sequences $\left(c_{k}(n)\right)_{k \geq 0}$. In fact such sequences, in particular the $n=2$ case, have important applications in harmonic analysis, theory of stable holomorphic functions and combinatorics. We refer the interested reader to [45, $46]$ and references therein.

\subsection{A matrix approach}

A matrix representation of real Appell sequences $\left(p_{k}(x)\right)_{k \geq 0}$ developed in [32] relies on the matrix $H$ whose entries are given by

$$
(H)_{i j}= \begin{cases}i, & i=j+1 \\ 0, & \text { otherwise }, \quad i, j=0,1, \ldots\end{cases}
$$

In this context the role of $H$ is essentially that of a derivation matrix.

For the sake of handle Appell sequences in a closed form, the vector

$$
\mathbf{p}(x)=\left[p_{0}(x) p_{1}(x) \cdots p_{m}(x)\right]^{T},
$$

whose entries are the $m+1$ first terms of the Appell sequence $\left(p_{k}(x)\right)_{k \geq 0}$, is introduced. Also, $H$ is truncated in order to obtain a square matrix of order $m+1$. In this case, $H$ is a nilpotent matrix of degree $m+1$. 
The differential equation $\mathbf{p}^{\prime}(x)=H \mathbf{p}(x)$ is the matrix counterpart of (9) for the truncated Appell sequence $\left(p_{k}(x)\right)_{0 \leq k \leq m}$. Its general solution is

$$
\mathbf{p}(x)=e^{H x} \mathbf{p}(0) \equiv \mathcal{P}(x) \mathbf{p}(0),
$$

where $\mathcal{P}(x)$ is the well known generalized Pascal matrix defined by

$$
(\mathcal{P}(x))_{i j}=\left\{\begin{array}{ll}
\left(\begin{array}{l}
i \\
j
\end{array}\right) x^{i-j}, & i \geq j \\
0, & \text { otherwise, }
\end{array} \quad i, j=0,1, \ldots, m,\right.
$$

Note that by using the explicit representation (11), $\mathbf{p}(0)=\left[\begin{array}{llll}a_{0} & a_{1} & \cdots & a_{m}\end{array}\right]^{T}$, $a_{0} \neq 0$.

Aiming a matrix approach of Appell sequences in the hypercomplex context, multivariate homogeneous polynomials of the form (13) are considered. Such polynomials correspond to (11) by replacing of real variable $x$ by $x_{0}$, real part of the paravector $x=x_{0}+\underline{x}$, and the constant $a_{j}$ by $a_{j} \underline{x}^{j}$.

Denoting by $\mathbf{P}(x)$ and $\mathbf{P}(\underline{x})$ the vectors

$$
\mathbf{P}(x) \equiv \mathbf{P}\left(x_{0}, \underline{x}\right)=\left[P_{0}(x) P_{1}(x) \cdots P_{m}(x)\right]^{T},
$$

and

$$
\mathbf{P}(\underline{x}) \equiv \mathbf{P}(0, \underline{x})=\left[\begin{array}{lll}
a_{0} \underline{x}^{0} & a_{1} \underline{x}^{1} \cdots a_{m} \underline{x}^{m}
\end{array}\right]^{T},
$$

respectively, a matrix form of the truncated sequence $\left(P_{k}(x)\right)_{0 \leq k \leq m}$ is

$$
\mathbf{P}(x)=e^{H x_{0}} \mathbf{P}(\underline{x}) .
$$

Considering the vector $\xi(\underline{x})=\left[1 \underline{x}^{1} \cdots \underline{x}^{m}\right]^{T}$ and the diagonal matrix

$$
\mathcal{D}=\operatorname{diag}\left[a_{0} a_{1} \cdots a_{m}\right],
$$

an equivalent representation of (17) is

$$
\mathbf{P}(x)=e^{H x_{0}} \mathcal{D} \xi(\underline{x}) .
$$

The relation (see [4])

$$
\partial_{\underline{x}}\left(\underline{x}^{k}\right)= \begin{cases}-k \underline{x}^{k-1}, & k \text { even } \\ -(n+k-1) \underline{x}^{k-1}, & k \text { odd }\end{cases}
$$

allows to achieve in matrix form the action of $\partial_{x}$ on the vector $\xi(\underline{x})$. In fact, straightforward calculations lead to $\partial_{\underline{x}} \xi(\underline{x})=\tilde{H} \xi(\underline{x})$, where $\tilde{H}$ is the matrix defined by

$$
(\tilde{H})_{i j}= \begin{cases}-(n+i-1), & i=j+1 \text { and } j \text { even } \\ -i, & i=j+1 \text { and } j \text { odd } \\ 0, & \text { otherwise }, i, j=0,1, \ldots, m\end{cases}
$$

(cf. [41], Proposition 3.1). 
Theorem 14. Let $\mathcal{D}$ be the matrix defined in (18), with $a_{0} \neq 0$, and $H, \tilde{H}$ as defined in (15), (20), respectively. If $\mathbf{P}(x)$ is the vector (16), then the following conditions are equivalent:

1. $\mathbf{P}(x)$ is an Appell vector;

2. The matrix $\mathcal{D}$ verifies the relation $H \mathcal{D}+\mathcal{D} \tilde{H}=0$.

Proof. ( $1 \Rightarrow 2)$ Applying to both sides of (19) the hypercomplex differential operator we obtain

$$
\bar{\partial} \mathbf{P}(x)=\frac{1}{2} e^{H x_{0}}(H \mathcal{D}+\mathcal{D} \tilde{H}) \xi(\underline{x}) .
$$

The result holds from the monogeneity of the components of $\mathbf{P}(x)$.

$(2 \Rightarrow 1)$ Under the hypothesis, the monogeneity of the components of $\mathbf{P}(x)$ is obvious. Furthermore, since $H \mathcal{D}=-\mathcal{D} \tilde{H}$,

$$
\begin{aligned}
\partial \mathbf{P}(x) & =\frac{1}{2} e^{H x_{0}}(H \mathcal{D}-\mathcal{D} \tilde{H}) \xi(\underline{x}) \\
& =H e^{H x_{0}} \mathcal{D} \xi(\underline{x})=H \mathbf{P}(x),
\end{aligned}
$$

which is the corresponding matrix representation of (12) in Definition \%. This completes the proof of the theorem.

\subsection{Orthogonality}

Practical problems of Physics and Engineering require to deal with numerical approximations of monogenic functions, like for instance, solutions of complicated differential equations. In this sense, the construction of basis of monogenic functions, in particular, monogenic polynomials, plays a crucial role. As we have seen, there exists equivalence between the existence of a local Taylor series in terms of symmetric powers, hypercomplex derivability/differentiability and monogeneity of Clifford-valued functions. The Fourier expansion can be achieved as well if we construct an orthonormal basis. Unfortunately, a basis formed by the symmetric powers is not orthogonal in $L_{2}$ and the numerical costs for its orthonormalization are enormous for higher degrees. A different approach was followed in [38] in the case of 3 dimensions using the concept of Gelfand-Tsetlin bases. The generalization of this approach to arbitrary dimensions was done in [37] leading to an iterative process where the building blocks are the (in general) non-monogenic $\mathcal{C} \ell_{0, n}$-valued polynomials

$$
X_{n+1, j}^{(k-j)}(x)=F_{n+1, j}^{(k-j)}(x)+\frac{j+1}{n+2 j} F_{n+1, j-1}^{(k-j-1)}(x) \underline{x},
$$

where

$$
F_{n+1, j}^{(k-j)}(x)=\frac{(j+1)_{k-j}}{(n-1+2 j)_{k-j}}|x|^{k-j} C_{k-j}^{\frac{n-1}{2}+j}\left(\frac{x_{0}}{|x|}\right),
$$

with $F_{n+1, k+1}^{(-1)} \equiv 0, x=\left(x_{0}, \ldots, x_{n}\right) \in \mathbb{R}^{n+1},|$.$| is the usual Euclidean norm in$ $\mathbb{R}^{n+1}$, and $C_{m}^{\nu}$ is the Gegenbauer polynomial of degree $m$ and parameter $\nu \neq 0$. 
The resulting basis of orthogonal ${ }^{4}$ monogenic polynomials is formed by the polynomials

$$
f_{k, \mu}=X_{n+1, k_{n}}^{\left(k-k_{n}\right)} X_{n, k_{n-1}}^{\left(k_{n}-k_{n-1}\right)} \cdots X_{3, k_{2}}^{\left(k_{3}-k_{2}\right)} \zeta^{k_{2}},
$$

where $\zeta:=x_{1}-x_{2} e_{1} e_{2}$ and $\mu$ is an arbitrary sequence of integers $\left(k_{n+1}, k_{n}, \ldots, k_{2}\right)$ such that $k=k_{n+1} \geq k_{n} \geq \ldots \geq k_{2} \geq 0$.

Earlier, in the book [4], multiples of the building blocks (21) appeared when the CK-extension was applied to the vector-valued polynomials $\underline{x}^{k-j} P_{j}(\underline{x})$, where $P_{j}(\underline{x})$ are arbitrarily fixed monogenic polynomials of degree $j(j=0, \ldots, k)$. Since the resulting polynomials do not have the Appell property, an appropriate normalization constant was considered by the first three authors in the paper [40]. More explicitly, the CK-extension was applied to the polynomials

$$
c_{k, j}(n)\left(\begin{array}{c}
k \\
j
\end{array}\right) \underline{x}^{k-j} P_{j}(\underline{x}),
$$

where

$$
c_{k, j}(n)= \begin{cases}\frac{(k-j) ! !(n+2 j-2) ! !}{(n+k+j-1) ! !}, & \text { if } k, j \text { have different parities } \\ c_{k-1, j}(n), & \text { if } k, j \text { have the same parity }\end{cases}
$$

for $k \geq 1, j=0, \ldots, k$ and $c_{0,0}(n)=1$. The result is the sequence of orthogonal monogenic $\mathcal{A}_{n}$-valued Appell polynomials $\left(\widetilde{X}_{n+1, j}^{(k-j)}\right)_{k \in \mathbb{N}_{0}}$, where

$$
\widetilde{X}_{n+1, j}^{(k)}(x):=X_{n+1, j}^{(k-j)}(x) P_{j}(\underline{x}), \quad x \in \mathcal{A}_{n} .
$$

The (in general) non-monogenic polynomials $X_{n+1, j}^{(k-j)}$ can be built by a simple shift of the coefficients of the monogenic SAP studied in the subsection 8.1. In fact, the paper [40] shows that

Theorem 15. For all $k \in \mathbb{N}_{0}$ and each fixed $j(j=0, \ldots, k)$, it holds

$$
X_{n+1, j}^{(k-j)}(x)=\left(\begin{array}{c}
k \\
j
\end{array}\right) \mathcal{P}_{k-j}^{n+2 j}(x), \quad x \in \mathcal{A}_{n} .
$$

Notice that for a fixed $k$ and $j=0, X_{n+1,0}^{(k)}$ coincide exactly with the $\operatorname{SAP} \mathcal{P}_{k}^{n}$, constituting the only monogenic building block in the sequence defined by (22). This fact is reflected in the monogenic sequence $\left(\widetilde{X}_{n+1, j}^{(k-j)}\right)_{k \in \mathbb{N}_{0}}$ by the choice $P_{0}(\underline{x}) \equiv 1$.

As a consequence of Theorem 15, the polynomials $X_{n+1, j}^{(k-j)}(j=0, \ldots, k)$, explicitly given by (21), admit the simpler representation

$$
X_{n+1, j}^{(k-j)}(x)=\left(\begin{array}{c}
k \\
j
\end{array}\right) \sum_{s=0}^{k-j}\left(\begin{array}{c}
k-j \\
s
\end{array}\right) c_{s}(n+2 j) x_{0}^{k-j-s} \underline{x}^{s}, \quad x \in \mathcal{A}_{n},
$$

\footnotetext{
${ }^{4}$ The inner product in $L_{2}$ is given by $(f, g)_{\mathcal{C}_{0, n}}=\int_{B^{n+1}} \bar{f} g d \lambda^{n+1}$, where $\lambda^{n+1}$ is the Lebesgue measure and $B^{n+1}$ is the unit ball in $\mathbb{R}^{n+1}$.
} 
where $c_{s}(n+2 j)(s=0, \ldots k-j, j=0, \ldots, k)$ are given by (14) with $c_{0}(n)=1$.

As it is well known from the theory, any sequence of orthogonal polynomials satisfies a three-term recurrence relation and a second order differential equation. The immediate question arises: can we derive similar results for the orthogonal sequence of monogenic polynomials $\left(\widetilde{X}_{n+1, j}^{(k)}: j=0, \ldots, k\right)_{k \in \mathbb{N}_{0}}$ ? The affirmative answer was done in the paper [40] leading to the following results.

Theorem 16. For all $k \in \mathbb{N}_{0}$ and each fixed $j(j=0, \ldots, k)$, the monogenic polynomials $\widetilde{X}_{n+1, j}^{(k-j)}(x), x \in \mathcal{A}_{n}$ satisfy the three-term type recurrence

$$
\begin{gathered}
(n+k+1+j)(k+2-j) \widetilde{X}_{n+1, j}^{(k+2)}-\left[(n+2 k+2) x_{0}+\underline{x}\right](k+2) \widetilde{X}_{n+1, j}^{(k+1)} \\
+(k+2)(k+1)|x|^{2} \widetilde{X}_{n+1, j}^{(k)}=0, \\
\widetilde{X}_{n+1, j}^{(j)}=P_{j}(\underline{x}), \widetilde{X}_{n+1, j}^{(j+1)}=(j+1)\left(x_{0}+\frac{1}{n+2 j} \underline{x}\right) P_{j}(\underline{x}) .
\end{gathered}
$$

Notice that the appearance of a polynomial of second degree as coefficient of $\widetilde{X}_{n+1, j}^{(k)}$ instead of a constant, like in the classical case, is justified by the fact that we are dealing with homogeneous polynomials.

Theorem 17. For all $k \in \mathbb{N}_{0}$ and each fixed $j(j=0, \ldots, k)$, the monogenic polynomials $\widetilde{X}_{n+1, j}^{(k)}(x), x \in \mathcal{A}_{n}$, satisfy the second order differential equation

$$
|x|^{2} \partial^{2} y(x)-\left((n+2 k-2) x_{0}+\underline{x}\right) \partial y(x)+(n+k+j-1)(k-j) y(x)=0 .
$$

Acknowledgments. The work of the first, second and fourth authors was supported by Portuguese funds through the CIDMA - Center for Research and Development in Mathematics and Applications, and the Portuguese Foundation for Science and Technology ("FCT-Fundação para a Ciência e Tecnologia"), within project PEst-OE/MAT/UI4106/2013. The work of the second author was supported by Portuguese funds through the CMAT - Centre of Mathematics and FCT within the Project UID/MAT/00013/2013.

\section{References}

1. Stein, E. M., Weiss, G.: Generalization of the Cauchy-Riemann Equations and Representations of the Rotation Group. American Journal of Mathematics 90 (1), 163-196 (1968)

2. Brackx, F., Delanghe, R., De Schepper, H.: Hardy spaces of solutions of generalized Riesz and Moisil-Teodorescu systems. Complex Var. Elliptic Equ. 57(7-8), 771-785 (2012).

3. G. Moisil and N. Teodorescu: Functions holomorphes dans l'espace. Mathematica Cluj, 5, 142-159 (1931)

4. Delanghe, R., Sommen, F., Souček, V.: Clifford Algebra and Spinor-Valued Functions. A Function Theory for the Dirac Operator. Mathematics and its Applications, vol. 53. Kluwer Academic Publishers, Dordrecht (1992) 
5. Delanghe, R., Lávička, R., Souček, V.: The Gelfand - Tsetlin bases for Hodge de Rham systems in Euclidean spaces Math. Methods Appl. Sci., 35(7) 745-757 (2012)

6. Delanghe, R.: On Regular-analytic Functions with Values in a Clifford Algebra. Math. Ann. 185, 91-111 (1970)

7. Kravchenko, V. V.: Applied quaternionic analysis. Research and Exp. in Mathematics, Vol. 28, Heldermann Verlag, Lemgo, (2003)

8. Delanghe, R.: Clifford Analysis: History and Perspective. Comput. Methods Funct. Theory 1(1), 107-153 (2001)

9. Fueter, R.: Über die analytische Darstellung der regulären Funktionen einer Quaternionenvariablen Comm. Math. Helvetici, 8, 371-378 (1935) https://doi.org/10.1007/BF01199562

10. Fueter, R.: Über Funktionen einer Quaternionenvariablen. Atti Congr. Int. Mat., Bologna (1928)

11. Malonek, H.: Rudolf Fueter and his motivation for hypercomplex function theory. Adv. Appl. Clifford Algebr. 11 (S2), 219-230 (2001)

12. Fueter, R.: Functions of a hyper complex variable. Manuscript of Lecture Notes, Fall Semester 1948/49, written and supplemented by E. Bareiss, ETH Bibliothek Zürich, 318p. (1950)

13. Gürlebeck, K., Habetha, K., Sprößig, W.: Holomorphic functions in the plane and $n$-dimensional space. Birkhäuser Verlag, Basel (2008)

14. Gürlebeck, K., Sprößig, W.: Quaternionic analysis and elliptic boundary value problems. International Series of Numerical Mathematics, vol.89, BirkhäuserVerlag, Basel (1990)

15. Ryan, J.: Clifford analysis with generalize elliptic and quasi-elliptic functions. Appl. Anal. 13, 151-171 (1982)

16. Brackx, F., Delanghe, R., Sommen, F.: Clifford Analysis. Pitman, Boston-LondonMelbourne (1982)

17. Malonek, H.: A new hypercomplex structure of the Euclidean space $\mathbb{R}^{n+1}$ and a concept of hypercomplex differentiability. Complex Variables, Theory Appl. 14, 25-33 (1990)

18. Malonek, H.: The concept of hypercomplex differentiability and related differential forms. In: Kühnau, R., Tutschke, W. (eds.) Studies in complex analysis and its applications to partial differential equations 1, 256 Pitman , Longman, pp. 193$202(1991)$

19. Malonek, H.: Hypercomplex derivability - The characterization of monogenic functions in $\mathbb{R}^{n+1}$ by their derivative. In: Ryan, J. et al. (eds.) Clifford Algebras and their Applications in Mathematical Physics, Vol. 2, Progress in Physics, Vol.19, Birkhäuser (2000)

20. Malonek, H.R.: Selected topics in hypercomplex function theory. In: Eriksson, S.L. (ed.), Clifford Algebras and Potential Theory. Report Series 7, University of Joensuu, pp. 111-150 (2004)

21. Gürlebeck, K., Malonek, H.: A hypercomplex derivative of monogenic functions in $\mathbb{R}^{n+1}$ and its applications. Complex Variables, Theory Appl. 39, 199-228 (1999)

22. Ryan, J.: Clifford Algebras in Analysis and Related Topics. Studies in Advanced Mathematics, CRC PRESS (1996)

23. Pompeiu, M.: Sur une classe de fonctions dúne variable complexe. Rend. Circ. Mat. Palermo 33, 108- 113 (1912), 35, 277-281 (1913)

24. Appell, P.: Sur une classe de polynômes. Ann. Sci. École Norm. Sup. 9(2),119-144 (1880) 
25. Mitrea, M., Sabac, F.: Pompeiu's integral representation formula. History and Mathematics. Rev. Roumaine Math. Pures Appl. 43, 211-226 (1998)

26. Edwards, H.E.: Advanced Calculus: A differential forms approach. 3rd ed., Birkhäuser, Boston - Basel - Berlin (1993)

27. Malonek, H.: On the concept of holomorphy in higher dimensions - Hypercomplex differentiability and series in permutative powers. Habilitation Thesis, Halle (1987)

28. Sudbery, A.: Quaternionic analysis. Math. Proc. Camb. Phil. Soc. 85, 199-225 (1979)

29. Malonek, H.: Power series representation for monogenic functions in $\mathbb{R}^{n+1}$ basead on a permutational product. Complex Variables, Theory Appl. 15, 181-191 (1990)

30. Sheffer, I.M.: Note on Appell polynomials. Bull. Amer. Math. Soc. 51, 739-744 (1945)

31. Lee, D.W.: On multiple Appell polynomials. Proc. Amer. Math. Soc. 139(6), 2133$2141(2011)$

32. Aceto, L., Malonek, H.R., Tomaz, G.: A unified matrix approach to the representation of Appell polynomials. Integral Transf. Spec. Funct. 26(6), 426-441 (2015)

33. Malonek, H.R., Falcão, M.I.: Special monogenic polynomials - properties and applications. In: Simos, T. S., Psihoyios, G., Tsitouras, Ch. (eds.) AIP Conference Proceedings, vol.936, pp. 765-767 (2007)

34. Cação I., Falcão, M.I., Malonek, H.R.: Laguerre derivative and monogenic Laguerre polynomials: An operational approach. Math. Comput. Model. 53(5-6), 1084-1094 (2011)

35. Bock, S., Gürlebeck, K.: On a generalized Appell system and monogenic power series. Math. Methods Appl. Sci. 33(4), 394-411 (2010)

36. Eelbode, D.: Monogenic Appell sets as representations of the Heisenberg algebra. Adv. Appl. Clifford Algebra 22(4), 1009-1023 (2012)

37. Làvička, R.: Complete orthogonal Appell systems for spherical monogenics. Complex Anal. Oper. Theory 6, 477-489 (2012)

38. Bock, S., Gürlebeck, K., Làvička, R., Souček, V.: Gelfand-Tstelin bases for spherical monogenics in dimension 3. Rev. Mat. Iberuom. 28(4), 1165-1192 (2012)

39. Cação, I., Falcão, M.I., Malonek, H.R.: Matrix representations of a basic polynomial sequence in arbitrary dimension. Comput. Methods Funct. Theory 12(2), 371-391 (2012)

40. Cação, I., Falcão, M.I., Malonek, H.R.: Three term recurrence relations for systems of Clifford algebra-valued polynomial sequences. Adv. Appl. Clifford Algebras 27, 71-85 (2017)

41. Aceto, L., Malonek, H.R., Tomaz, G.: Matrix approach to hypercomplex Appell polynomials. Appl. Numer. Math. 116, 2-9 (2017)

42. Cação, I., Falcão, M.I., Malonek, H.R.: Combinatorial Identities in the Context of Hypercomplex Function Theory. AIP Conf. Proc. 1978, 280004-1-280004-4; https://doi.org/10.1063/1.5043904 (2018)

43. Cruz, C., Falcão, M.I., Malonek, H.R.: Monogenic pseudo-complex power functions and their applications. Math. Methods Appl. Sci. 37, 1723-1735 (2014)

44. Peña Peña, D.: Shifted Appell Sequences in Clifford Analysis. Results Math. 63, 1145-1157 (2013)

45. Cação, I., Falcão, M.I., Malonek, H.R.: Hypercomplex polynomials, Vietoris' rational numbers and a related integer numbers sequence. Complex Anal. Oper. Theory 11, 1059-1076 (2017)

46. Cação, I., Falcão, M.I., Malonek, H.R., Tomaz, G.: Combinatorial identities associated with a multidimensional polynomial sequence. J. Integer Seq. 21(7), 18.7.4 (2018) 